Article

\title{
Hypolipidemic Effects and Safety of Lactobacillus Reuteri 263 in a Hamster Model of Hyperlipidemia
}

\section{Wen-Ching Huang ${ }^{1, \dagger}$, Yi-Ming Chen ${ }^{2, \dagger}$, Nai-Wen Kan ${ }^{1,3}$, Chun-Sheng Ho ${ }^{4,5}$, Li Wei ${ }^{6}$, Ching-Hung Chan ${ }^{7}$, Hui-Yu Huang ${ }^{7, *}$ and Chi-Chang Huang ${ }^{2,4, *}$}

1 Graduate Institute of Athletics and Coaching Science, National Taiwan Sport University, Taoyuan 33301, Taiwan; E-Mails: 1010503@ntsu.edu.tw (W.-C.H.); kevinkan@tmu.edu.tw (N.-W.K.)

2 Graduate Institute of Sports Science, National Taiwan Sport University, Taoyuan 33301, Taiwan; E-Mail: 1021302@ntsu.edu.tw

3 Center for Liberal Arts, Taipei Medical University, Taipei 11031, Taiwan

4 College of Exercise and Health Sciences, National Taiwan Sport University, Taoyuan 33301, Taiwan; E-Mail: 1031213@ntsu.edu.tw

5 Division of Physical Medicine and Rehabilitation, Lo-Hsu foundation, Inc., Lotung Poh-Ai Hospital, Yilan 26546, Taiwan

6 Department of Neurosurgery, Taipei Medical University-WanFang Hospital, Taipei 11696, Taiwan; E-Mail: nsweili@gmail.com

7 Department of Food Science, Nutrition, and Nutraceutical Biotechnology, Shih Chien University, Taipei 10462, Taiwan; E-Mail: 1lfonly_520@hotmail.com

$\dagger$ These authors contributed equally to this work.

* Authors to whom correspondence should be addressed; E-Mails: maggieh@g2.usc.edu.tw (H.-Y.H.); john5523@ntsu.edu.tw (C.-C.H.); Tel.: +886-2-2538-1111 (ext. 6223) (H.-Y.H.); +886-3-328-3201 (ext. 2409) (C.-C.H.).

Received: 11 March 2015 / Accepted: 12 May 2015 / Published: 15 May 2015

\begin{abstract}
We aimed to verify the beneficial effects of probiotic strain Lactobacillus reuteri 263 (Lr263) on hypolipidemic action in hamsters with hyperlipidemia induced by a $0.2 \%$ cholesterol and 10\% lard diet (i.e., high-cholesterol diet (HCD)). Male Golden Syrian hamsters were randomly divided into two groups: normal $(n=8)$, standard diet (control), and experimental $(n=32)$, a HCD. After a two-week induction followed by a six-week supplementation with Lr263, the 32 hyperlipidemic hamsters were divided into four groups ( $n=8$ per group) to receive vehicle or $\operatorname{Lr} 263$ by oral gavage at $2.1,4.2$, or $10.5 \times 10^{9}$ cells $/ \mathrm{kg} /$ day
\end{abstract}


for 6 weeks, designated the HCD, 1X, 2X and 5X groups, respectively. The efficacy and safety of Lr263 supplementation were evaluated by lipid profiles of serum, liver and feces and by clinical biochemistry and histopathology. HCD significantly increased serum levels of total cholesterol (TC), triacylglycerol (TG) cholesterol, high-density lipoprotein cholesterol (HDL-C), and low-density lipoprotein cholesterol (LDL-C), LDL-C/HDL-C ratio, hepatic and fetal TC and TG levels, and degree of fatty liver as compared with controls. Lr263 supplementation dose dependently increased serum HDL-C level and decreased serum TC, TG, LDL-C levels, LDL-C/HDL-C ratio, hepatic TC and TG levels, and fecal TG level. In addition, Lr263 supplementation had few subchronic toxic effects. Lr263 could be a potential agent with a hypolipidemic pharmacological effect.

Keywords: hypolipidemic; cholesterol; triglyceride; high-cholesterol diet; lipid-lowering

\section{Introduction}

Hyperlipidemia is a widely known key risk factor for cardiovascular diseases. High blood cholesterol and triacylglycerol levels are commonly considered important modulators and biomarkers of hyperlipidemic processes [1]. Therefore, the management of these two parameters is necessary for cardiovascular health. Probiotic bacteria are defined by the World Health Organization (WHO) as "live microorganisms which when administered in adequate amounts confer a health benefit on the host" and are being examined for their efficacy in lowering total cholesterol (TC) and low-density lipoprotein cholesterol (LDL-C) in humans [2]. Intestinal lactic acid bacterial (LAB) species with alleged health beneficial properties have been introduced as probiotics. LAB species are important members of the normal intestinal microflora and showed beneficial effects in study of the molecular biology and genomics of Lactobacillus in immune function, anti-cancer, and antibiotic-associated diarrhea, travelers' diarrhea, pediatric diarrhea, inflammatory bowel disease and irritable bowel syndrome [3].

Lactobacillus spp. occurs in the gastrointestinal ecosystem of humans, poultry, swine, and other animals. They are excellent probiotic microorganisms because of their activities in ameliorating enteric diseases, maintaining health, and inhibiting melanin synthesis [4,5]. Lactobacillus reuteri produces a broad-spectrum antimicrobial substance during fermentation of glycerol, which revealed that glycerol fermentation was associated with the production of beta-hydroxypropionic acid and trimethylene glycol [6]. L. reuteri is used as a probiotic for chronic constipation [7], inhibits Helicobacter pylori load in humans [8], and removes cholesterol [2].

Previous studies used the hamster model to evaluate the hypolipidemic effect because it has many similarities with human fat-induced atherosclerotic disease. As for humans, hamsters are endowed with cholesterol ester transfer protein and all of the enzymatic pathways in lipoproteins and bile metabolism; atherosclerotic plaques develop in response to a fat diet in lesion-prone areas similar to humans [9-11].

L. reuteri 263 is a patented strain for improving the syndrome of diabetes (US 20110300117 A1) and renal fibrosis in diabetes (US 20120183504 A1), which is different from other strains such as L. reuteri L3 for preventing obesity in obese mice [12] or the L. reuteri LR6-fermented product for controlling hyperlipidemia in rats [13]. In addition, species of the same bacterial strains or even strains of the same 
species may feature different biological functions [12]. Qiao et al. demonstrated that L. reuteri L3 but not $L$. reuteri L10 had anti-inflammation and anti-obesity properties for obese mice [12]. Because of the complexity of host-bacterial cross-talk and the importance of investigating specific bacterial strains, we conducted experiments to evaluate the therapeutic effectiveness of L. reuteri 263 supplementation on the regulation of hyperlipidemia in a dyslipidemic hamster model. We also examined the biochemical parameters and liver tissues by histopathology.

\section{Experimental Section}

\subsection{Materials, Animals, and Experiment Design}

L. reuteri 263 (Lr263) was obtained from GenMont Biotech Inc. (Tainan, Taiwan). The dose of Lr263 for humans is $900 \mathrm{mg}$ per day (lyophilized powder), equivalent to a daily recommended dose at $2.1 \times 10^{9}$ cells/serving/day. The hamster dose $(111 \mathrm{mg} / \mathrm{kg})$ was converted from a human equivalent dose (HED) based on body surface area by the following formula from the US Food and Drug Administration: assuming a human weight of $60 \mathrm{~kg}$, the HED for $900(\mathrm{mg}) / 60(\mathrm{~kg})=15 \times 7.4=111 \mathrm{mg} / \mathrm{kg}$; the conversion coefficient 7.4 was used to account for differences in body surface area between hamster and human as we recently described [14].

Specific pathogen-free male Golden Syrian hamsters (12 weeks old) were purchased from the National Laboratory Animal Center, Taipei City, Taiwan. Animals were housed in the animal facility at National Taiwan Sport University at room temperature $\left(22 \pm 1{ }^{\circ} \mathrm{C}\right)$ and $50 \%$ to $60 \%$ relative humidity, with a $12 \mathrm{~h}$ light-dark cycle (light on 7:00 AM). Distilled water and standard laboratory chow diet (No. 5001; PMI Nutrition International, Brentwood, MO, USA) were provided ad libitum. Before the experiments, the hamsters were acclimatized for 1 week to the environment and diet. The Institutional Animal Care and Use Committee (IACUC) of National Taiwan Sport University (NTSU) approved all animal experimental protocols, and the study conformed to the guidelines of the protocol IACUC-10307 approved by the IACUC ethics committee.

The experimental design is in Figure 1. After one-week acclimatization, 40 hamsters were divided randomly into two groups: normal $(n=8)$, fed a standard chow diet (control), and experimental $(n=32)$, fed a high-cholesterol diet (HCD) containing $0.2 \%$ cholesterol and $10 \%$ lard diet. The 32 resulting hyperlipidemic hamsters were divided into four groups ( $n=8$ /each group): (1) HCD with vehicle (water) treatment (HCD); (2) HCD with $111 \mathrm{mg} / \mathrm{kg} \mathrm{Lr} 263$ (Lr263-1X); (3) HCD with $222 \mathrm{mg} / \mathrm{kg} \mathrm{Lr263} \mathrm{(Lr263-2X);}$ (4) HCD with $555 \mathrm{mg} / \mathrm{kg}$ Lr263 (Lr263-5X). The vehicle group received the same volume of solution equivalent to body weight (BW). The food intake and water consumption were monitored daily, and BW was recorded weekly. 


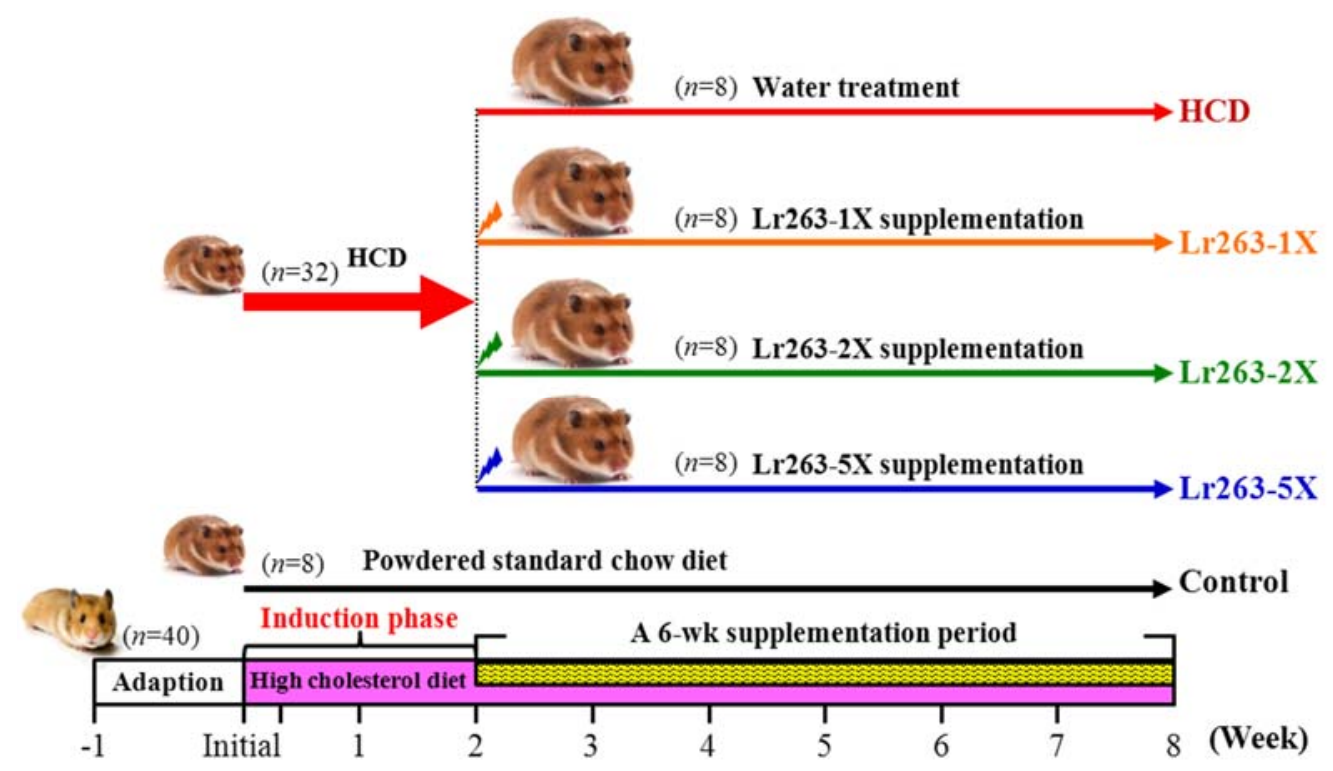

Figure 1. Experimental design. Control: Healthy hamsters were fed a standard laboratory diet and orally received the same volume of solution equivalent to body weight (BW). HCD: Hyperlipidemic hamsters were fed a high-cholesterol diet (HCD) and orally received the same volume of solution equivalent to BW. Lr263-1X: Hyperlipidemic hamsters were fed an HCD and orally received $111 \mathrm{mg} / \mathrm{kg}$ /day Lr263. Lr263-2X: Hyperlipidemic hamsters were fed an HCD and orally received $222 \mathrm{mg} / \mathrm{kg} /$ day Lr263. Lr263-5X: Hyperlipidemic hamsters were fed an HCD and orally received $555 \mathrm{mg} / \mathrm{kg} /$ day $\mathrm{Lr} 263$.

\subsection{HCD Composition}

Hamsters were fed a standard chow diet or an HCD adapted from previous study [15] with some modification. The standard chow (No. 5001) contained $3.35 \mathrm{kcal} / \mathrm{g}$ with $28.5 \%$ as protein, $13.5 \%$ as fat and $58.0 \%$ as carbohydrates. The HCD contained $0.2 \%$ (wt/wt) cholesterol (Sigma-Aldrich, St. Louis, MO, USA), 10\% (wt/wt) lard (Sigma-Aldrich) and 89.8\% (wt/wt) standard chow, for $3.92 \mathrm{kcal} / \mathrm{g}$ with $21.96 \%$ as protein, $33.37 \%$ as fat and $44.67 \%$ as carbohydrates.

\subsection{Liver and Fecal Lipid Analysis}

Liver and fecal matter were collected after hamsters were killed. Hepatic and fecal TG and TC levels were measured in triplicate by using commercial enzymatic kits for TG (No. 10010303) and for TC (No. 10007640) from Cayman Chemical (Ann Arbor, MI, USA).

\subsection{Clinical Biochemical Profiles}

At the end of the experimental period, all hamsters were killed with $95 \% \mathrm{CO}_{2}$ asphyxiation, and blood was immediately collected. Serum was collected by centrifugation and the clinical biochemical variables including aspartate aminotransferase (AST), alanine aminotransferase (ALT), lactate dehydrogenase (LDH), total protein (TP), blood urea nitrogen (BUN), creatinine, and glucose were measured by use of an autoanalyzer (Hitachi 7060, Tokyo, Japan). 


\subsection{Histological Staining of Tissues}

Liver tissues were carefully removed, minced and fixed in 10\% formalin. All samples were embedded in paraffin and cut into $4-\mu \mathrm{m}$ thick slices for morphological and pathological evaluations. Tissue sections were stained with hematoxylin and eosin (H\&E) and examined under a light microscope equipped with a CCD camera (BX-51, Olympus, Tokyo, Japan) by a veterinary pathologist.

\subsection{Statistical Analysis}

All data are expressed as mean \pm SD. Statistical differences were analyzed by one-way ANOVA and the Cochran-Armitage test for trend analysis of dose-effect of Lr263 supplementation with use of SAS 9.0 (SAS Inst., Cary, NC, USA). $p<0.05$ was considered statistically significant.

\section{Results and Discussion}

\subsection{Hamster BW and Daily Intake}

The growth curves for hamsters are in Figure 2. In the adaption and induction phase, BW was stable and steadily increased in each group. Hyperlipidemic and control hamsters did not differ in BW at the initial and induction phases and the end of the experiment. Therefore, the HCD did not affect BW. With Lr263 supplementation, the BW curve was still stable and steadily increased, with no significant differences among groups.

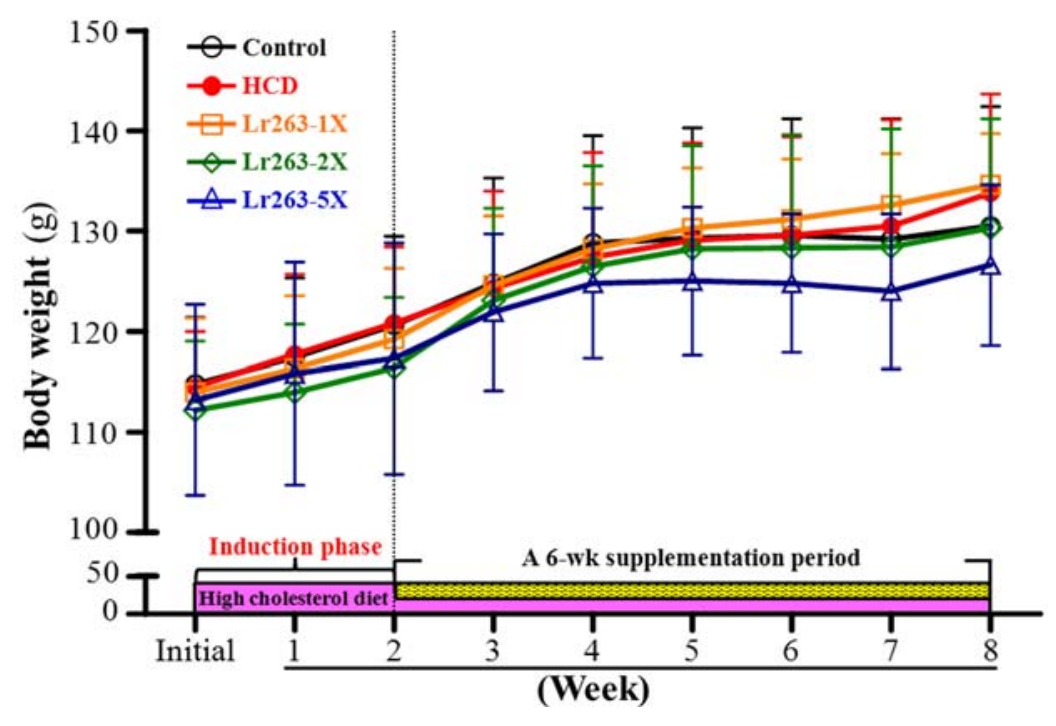

Figure 2. Change in BW during the experiment. The first week was an adaption phase. At two weeks, animals were separated into the control group and fed a standard laboratory diet (eight hamsters) or experimental animals and fed an HCD of the standard laboratory diet supplemented with $0.2 \%$ cholesterol and $10 \%$ lard (32 hamsters). Serum triglycerides (TG) and total cholesterol (TC) levels were higher for HCD than control hamsters. The 32 hamsters were randomly assigned to three groups (eight hamsters/group) for Lr263 supplementation. Data are mean $\pm \mathrm{SD}, n=8$ per group. 
The daily intake of hamsters is in Table 1 . The groups did not differ in intake in the adaption and induction phases and Lr263 supplementation period. Thus, BW and daily intake of hamsters increased normally and did not differ among the groups.

Table 1. Body weight (BW) and daily food intake for the experimental groups.

\begin{tabular}{|c|c|c|c|c|c|c|}
\hline Characteristics & Control & HCD & Lr263-1X & Lr263-2X & Lr263-5X & $\begin{array}{c}\text { Trend } \\
\text { Analysis }\end{array}$ \\
\hline Initial BW (g) & $115 \pm 7$ & $114 \pm 6$ & $114 \pm 7$ & $112 \pm 7$ & $113 \pm 9$ & 0.7344 \\
\hline Final BW (g) & $130 \pm 12$ & $134 \pm 10$ & $135 \pm 5$ & $130 \pm 11$ & $127 \pm 8$ & 0.0195 \\
\hline \multicolumn{7}{|l|}{ Adaption phase } \\
\hline $\begin{array}{c}\text { Deit intake } \\
\text { (g/hamster/day) }\end{array}$ & $8.02 \pm 0.75$ & $7.95 \pm 1.04$ & $8.00 \pm 0.73$ & $7.99 \pm 0.69$ & $8.05 \pm 0.52$ & 0.8111 \\
\hline Induction phase & & & & & & \\
\hline $\begin{array}{c}\text { Deit intake } \\
\text { (g/hamster/day) }\end{array}$ & $9.08 \pm 0.19$ & $9.04 \pm 0.45$ & $9.09 \pm 0.35$ & $9.09 \pm 0.32$ & $9.01 \pm 0.33$ & 0.8223 \\
\hline $\begin{array}{c}\text { Lr263 } \\
\text { Supplementation } \\
\text { Deit intake } \\
\text { (g/hamster/day) }\end{array}$ & $8.06 \pm 0.68$ & $8.23 \pm 0.88$ & $8.06 \pm 0.94$ & $8.13 \pm 0.75$ & $8.22 \pm 0.68$ & 0.8348 \\
\hline
\end{tabular}

Data are mean \pm SD, $n=8$ hamsters in each group. HCD: Hyperlipidemic hamsters fed a high-cholesterol diet (HCD) and orally received the same volume of solution equivalent to BW; Lr263-1X: Hyperlipidemic hamsters fed an HCD and orally received $111 \mathrm{mg} / \mathrm{kg}$ /day Lactobacillus reuteri 263 (Lr263). Lr263-2X: Hyperlipidemic hamsters fed an HCD and orally received $222 \mathrm{mg} / \mathrm{kg} /$ day Lr263. Lr263-5X: Hyperlipidemic hamsters fed an HCD and orally received $555 \mathrm{mg} / \mathrm{kg} /$ day $\operatorname{Lr} 263$.

\subsection{Effect of Two-Week HCD Induction on Serum TC and TG Levels}

Serum TG and TC levels in the two weeks after induction significantly differed among groups $(\mathrm{F}(4,35)=7.68, p<0.05, \eta 2=0.468 ; \mathrm{F}(4,35)=18.61, p<0.05, \eta 2=0.68$, respectively) (Figure 3). TG and TC levels were higher with the HCD alone (before Lr263 treatment), by 1.98- to 2.18-fold $(p<0.0005)$, than controls. Supplementation with an HCD for two weeks could significantly increase the serum TC and TG levels, for an animal model of hyperlipidemia.

The mouse, rat, golden hamster, guinea pig, rabbit, pigeon and quail are often used for a hyperlipidemia disease model. Previous studies have shown that the hamster model is similar to humans in lipid metabolism (e.g., in synthesis and secretion of cholesterol). The model possesses superior efficacy in preclinical evaluation, whereas in models of rats, mice, pigeons and quails, lipoprotein metabolism differs from that in humans $[16,17]$. Hamsters may be a better animal model for hypercholesterolemia because the content of hyperlipidemia is easily maintained with high-fat, HCD induction $[10,18]$. Therefore, the hamster model has been often used to study hyperlipidemia [19-21]. 
A

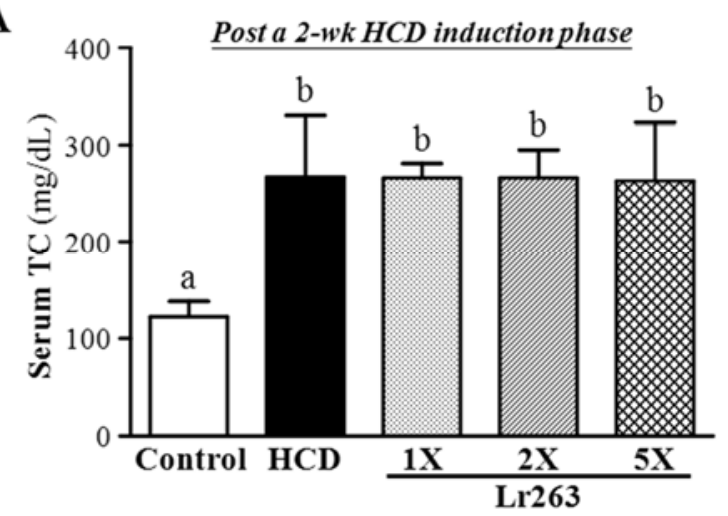

B

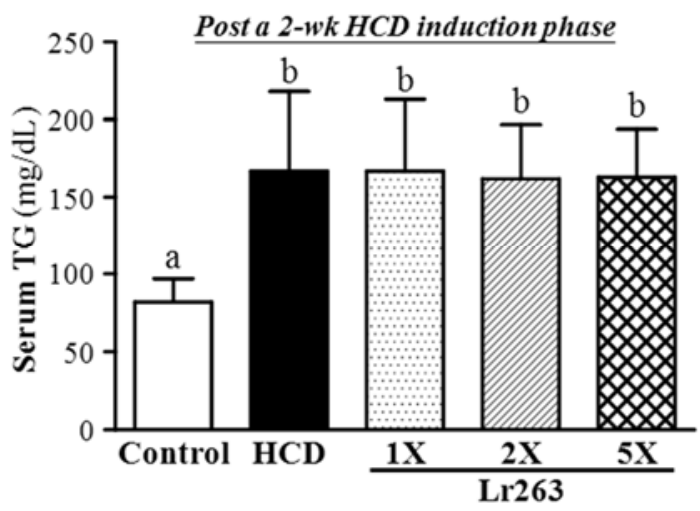

Figure 3. Effect of two-week HCD on serum levels of TC (A) and TG (B) in hamsters. Data are the mean $\pm \mathrm{SD}, n=8$ hamsters in each group. Columns with different letters $(\mathrm{a}, \mathrm{b})$ significantly differ at $p<0.05$ by a one-way ANOVA.

\subsection{Effect of Six-Week Supplementation with Lr263 on Serum Lipid Levels and LDL-C/HDL-C Ratio in Hyperlipidemic Hamsters}

At six weeks after Lr263 supplementation, TC levels significantly differed among groups $(\mathrm{F}(4,35)=58.75, p<0.05, \eta 2=0.87)$ (Figure 4A). TC levels were lower with Lr263-1X, Lr263-2X and Lr263-5X (18.6\% $(p=0.0003), 19.5 \%(p=0.0002)$ and 23.1\% $(p<0.0001)$, respectively) than with HCD alone. Serum TG levels differed among groups $(\mathrm{F}(4,35)=21.60, p<0.05, \eta 2=0.712)$, with levels higher for the HCD group, by 3.15-fold ( $p<0.0001$ ), than controls (Figure 4B). However, TG levels were lower with Lr263-1X, Lr263-2X and Lr263-5X [42.4\% $(p<0.0001), 40.6 \%(p<0.0001)$ and $45.9 \%(p<0.0001)$, respectively] than with HCD alone. On trend analysis, TC and TG content was increased dose-dependently with Lr263 treatments under HCD-induced hyperlipidemia. Therefore, Lr263 can reduce serum TC and TG levels in the hyperlipidemic hamster L. reuteri during fermentation to produce glycerol. A previous study found a relationship between glycerol release and periovarian fat weight; the authors demonstrated that glycerol release decreased with increased blood free fatty acid, TG, and insulin [22]. In relevant Lactobacillus spp. supplementation studies, oral administration of probiotics significantly lowered cholesterol levels by as much as $22 \%$ to $33 \%$ in humans [23] or prevented elevated cholesterol levels in mice fed a fat-enriched diet [24].

At the end of the experiment, the high-density lipoprotein cholesterol (HDL-C) levels were significantly different among groups $(\mathrm{F}(4,35)=14.39, p<0.05, \eta 2=0.622)$ (Figure 4C,D). HDL-C levels were higher with HCD alone and with $1 \mathrm{X}, 2 \mathrm{X}$, and $5 \mathrm{X}$ Lr263, by $1.38-(p=0.0004), 1.51$ $(p<0.0001), 1.62-(p<0.0001)$ and 1.62-fold $(p<0.0001)$ than controls. Furthermore, HDL-C levels were higher with $2 \mathrm{X}$ and $5 \mathrm{X} \operatorname{Lr} 263$ than $\operatorname{HCD}$ alone $(p=0.0153$ and $p=0.0175$, respectively). In addition, serum LDL-C levels differed among groups $(\mathrm{F}(4,35)=14.92, p<0.05, \eta 2=0.63)$. LDL-C levels were lower with HCD combined with Lr263-1X, Lr263-2X and Lr263-5X treatments (33\% $(p<0.0001), 48.7 \%(p<0.0001)$, and 49.1\% $(p=0.0023)$, respectively) than HCD alone. On trend analysis, HDL-C and LDL-C was dose-dependently altered with Lr263 supplementation $(p=0.003$ and $p<0.0001$, respectively). In a previous study, an HCD could enhance serum HDL-C and LDL-C levels in the same hamster model [25]. Thus, LR263 could have modulatory effects on LDL-C levels, for a potential pharmacological effect on hyperlipidemia. 
In a previous study, several mechanisms for cholesterol removal by Lactobacillus spp. have been proposed; one is deconjugation of bile salts by bile-salt hydrolase (BSH) assimilation of cholesterol into bacterial cell membranes to reduce cholesterol level [26]. The ability of probiotic strains to hydrolyze bile salts has often been included among the criteria for probiotic strain selection, and a number of BSHs have been identified and characterized [27]. Oral administration of Lactobacillus spp. was found to significantly reduce cholesterol levels, with no significant improvement in LDL-C/HDL-C ratio [28]. Therefore, different lactobacillus strains may have different cholesterol-lowering abilities.

The ratio of LDL-C/HDL-C is a criterion for evaluating the efficiency of cholesterol-lowering capacity. If the ratio is low, atherosclerotic risk factors are decreased [29]. The ratio of LDL-C/HDL-C calculated from individual hamsters differed among groups $(\mathrm{F}(4,35)=8.14, p<0.05, \eta 2=0.482)$ and was higher for HCD alone, by 5.47-fold ( $p<0.0001$ ), than controls (Figure 4E). The ratio of LDL-C/HDL-C was lower with LR263-1X, LR263-2X and LR263-5X [36.1\% $(p=0.0234), 58.0 \%(p=0.0005)$ and 58.1\% $(p=0.0005)$, respectively] than with HCD alone. On trend analysis, the ratio of LDL-C/HDL-C was dose-dependently decreased with Lr263 supplementation $(p<0.0001)$. In a previous study, a probiotic mix was found to modulate apolipoprotein synthesis. The mechanism was via a coordinated enterohepatic action that might be mediated by PPAR gamma/FXR upregulation [30]. In the current study, Lr263 could ameliorate cholesterol levels and improve the LDL-C/HDL-C ratio under HCD diet-induced hyperlipidemia in hamsters.

A

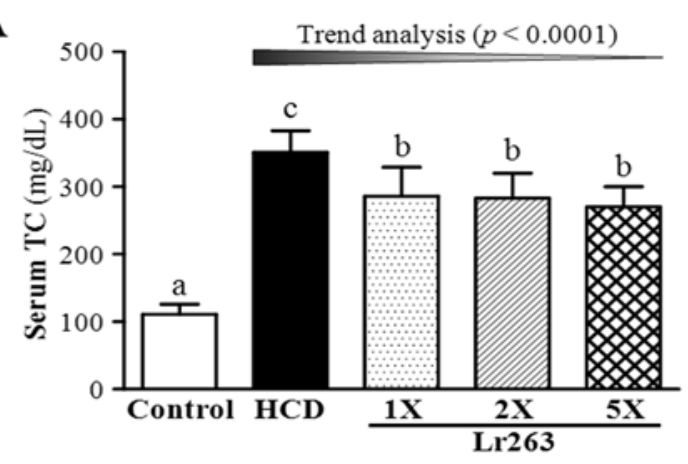

C

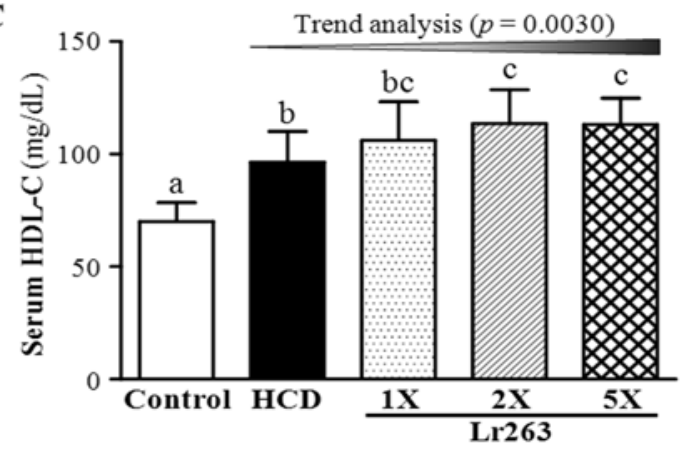

B

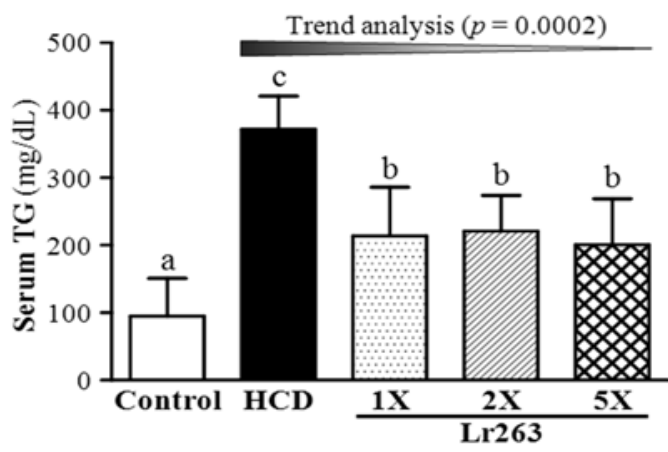

D

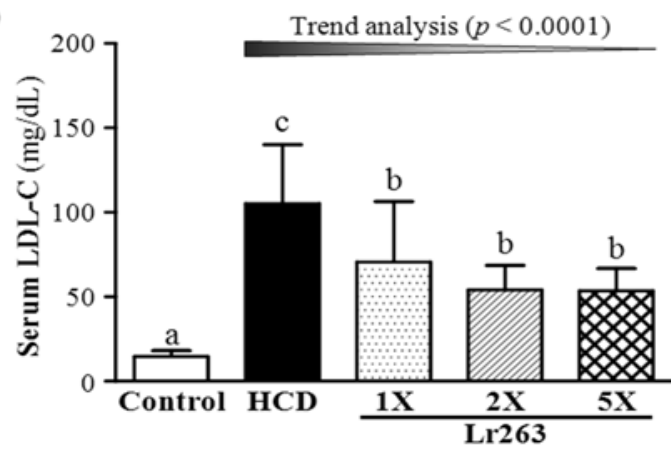

Figure 4. Cont. 


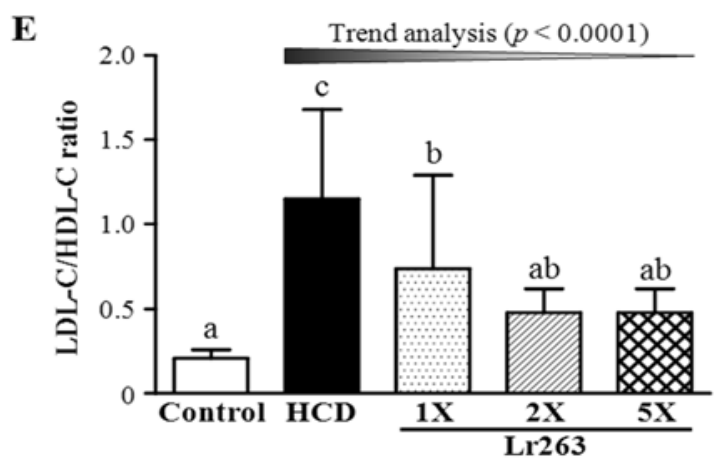

Figure 4. Effect of six-week supplementation with Lr263 on serum TC (A); TG (B); HDL-C (C); LDL-C (D) levels; and LDL-C/HDL-C ratio (E) in hyperlipidemic hamsters. Data are mean $\pm \mathrm{SD}, n-8$ hamsters in each group. Columns with different letters $(\mathrm{a}, \mathrm{b}, \mathrm{c})$ differ significantly at $p<0.05$ by a one-way ANOVA.

\subsection{Effect of Six-Week Supplementation with Lr263 on Hepatic TC and TG Levels in} Hyperlipidemic Hamsters

Liver TC content significantly differed among groups $(\mathrm{F}(4,35)=32.82, p<0.05, \eta 2=0.789)$ and was higher with HCD alone, by 2.67 -fold ( $p<0.0001$ ), than controls (Figure 5A). Furthermore, liver TC level was lower with Lr263-1X, Lr263-2X and Lr263-5X $(25.1 \%(p=0.0001), 22.2 \%(p=0.0005)$ and $39.0 \%(p<0.0001)$, respectively) than HCD alone.

Liver TG content was significantly different among groups $(\mathrm{F}(4,35)=9.59, p<0.05, \eta 2=0.523)$ and was higher with HCD, by 1.83 -fold $(p<0.0001)$, than controls (Figure 5B). Liver TG content was lower with Lr263-1X, Lr263-2X and Lr263-5X (30.3\% ( $p=0.0003), 26.1 \%(p=0.0014)$ and 26.9\% $(p<0.001)$, respectively) than HCD alone. On trend analysis, liver TC and TG content dose-dependently decreased with Lr263 supplementation ( $p<0.0001$ and $p=0.0365$, respectively). Lr263 supplementation could significantly mitigate the increased liver TC and TG content induced by the HCD hyperlipidemia model.
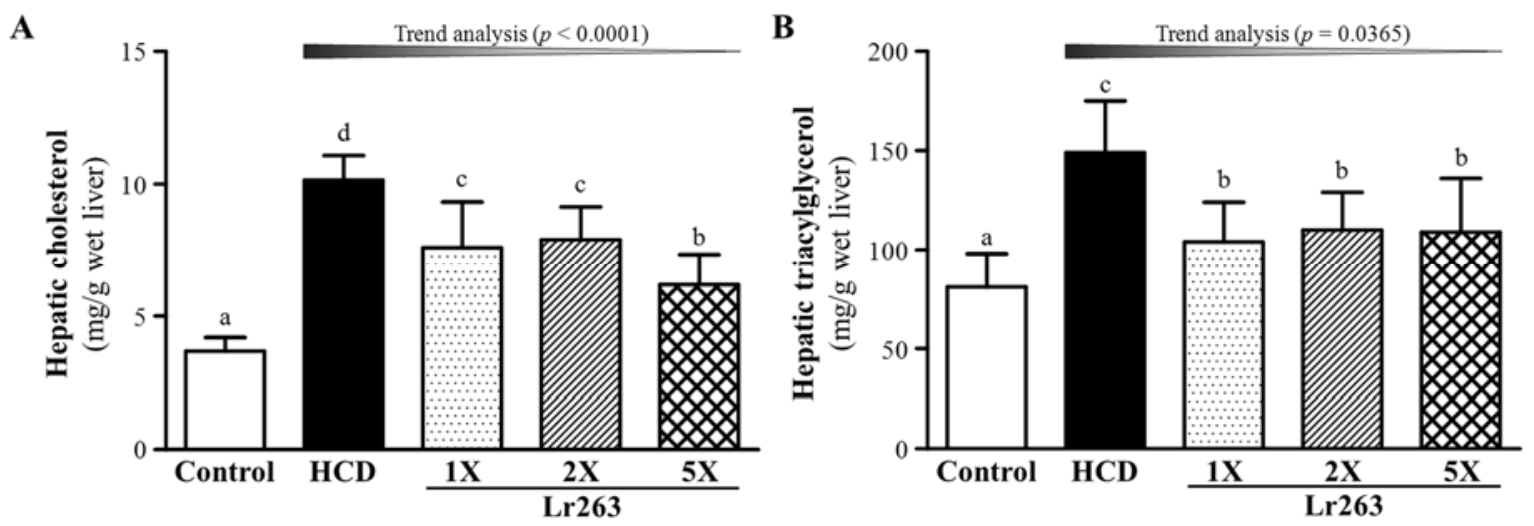

Figure 5. Effect of six-week supplementation with Lr263 on hepatic TC (A) and TG (B) levels in hyperlipidemic hamsters. Data are the mean $\pm \mathrm{SD}, n=8$ hamsters in each group. Columns with different letters $(\mathrm{a}, \mathrm{b}, \mathrm{c}, \mathrm{d})$ significantly differ at $p<0.05$ by a one-way ANOVA. 


\subsection{Effect of Six-Week Supplementation with Lr263 on Fecal TC and TG Levels in Hyperlipidemic Hamsters}

Fecal TC content differed among groups $(\mathrm{F}(4,35)=5.70, p<0.05, \eta 2=0.395)$ and was higher with HCD alone, by 1.42-fold ( $p=0.0003$ ), than controls (Figure 6A). Fecal TC content did not differ by Lr263 supplementation. Therefore, the HCD could significantly increase the fecal TC level in all cholesterol-treated groups.

The fecal TG levels significantly differed among groups $(\mathrm{F}(4,35)=9.95, p<0.05, \eta 2=0.532)$ and was higher with HCD alone, by 2.14-fold ( $p<0.0001$ ), than controls (Figure 6B). Furthermore, fecal TG content was lower with Lr263-1X, Lr263-2X and Lr263-5X, [23.7\% ( $p=0.0088), 22.1 \%(p=0.0141)$ and $23.5 \%(p=0.0092)$, respectively] than HCD alone. On trend analysis, Lr263 supplementation showed dose-dependently decreased fecal TG content $(p=0.0338)$. Therefore, our HCD could increase both serum and fecal TG levels. Lr263 treatment could manage excessive fecal and serum TG levels.

A

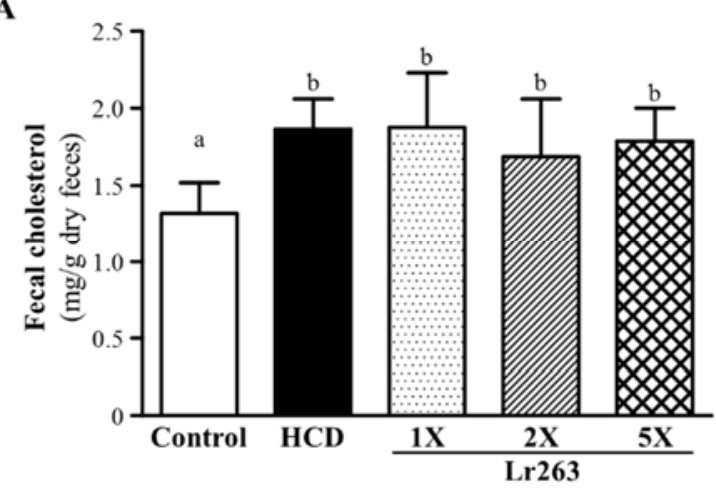

B

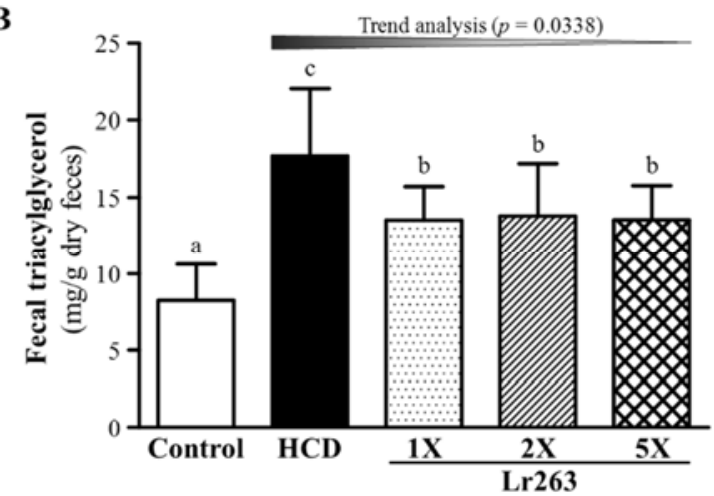

Figure 6. Effect of six-week supplementation with Lr263 on fecal TC (A) and TG (B) levels in hyperlipidemic hamsters. Data are mean $\pm \mathrm{SD}, n=8$ hamsters in each group. Columns with different letters $(\mathrm{a}, \mathrm{b}, \mathrm{c})$ significantly differ at $p<0.05$ by a one-way ANOVA.

\subsection{Effect of Lr263 Supplementation on Tissue Weight at the End of the Experiment}

Hamsters were killed after 6 weeks of Lr263 supplementation; liver, kidney, heart and epididymal fat pad (EFP) were removed and tissue weight was recorded for evaluating body composition. Kidney and heart weight and relative kidney and liver weight (\%) did not differ among groups (Table 2).

The liver and EFP weight significantly differed among groups $(\mathrm{F}(4,35)=66.07, p<0.05, \eta 2=0.883$; $\mathrm{F}(4,35)=4.22, p<0.05, \eta 2=0.325$, respectively) and was higher with HCD alone, by 1.71- and 1.40-fold $(p<0.0001)$, than controls. The liver weight was lower with Lr263-1X, Lr263-2X and Lr263-5X supplementation $[8.4 \%(p=0.0045), 8.4 \%(p=0.0047)$ and $10.4 \%(p=0.0006)$, respectively] than HCD alone. In a previous study, liver weight significantly increased with a high-cholesterol diet [31]. We found that Lr263 supplementation could decrease the increased liver weight caused by a high cholesterol diet. The EFP weight was lower with Lr263-1X, Lr263-2X and Lr263-5X (8.4\% ( $p=0.0045), 8.4 \%$ $(p=0.0047)$ and $10.4 \%(p=0.0006)$, respectively) than HCD alone. EFP weight was less with Lr263-2X and Lr263-5X (19.7\% $(p=0.0104)$ and 19.2\% ( $p=0.012)$, respectively) than HCD alone. A similar study, involving kimchi lactic acid bacteria, showed significantly reduced TG and TC levels in liver 
and epididymal adipose tissue in a high-cholesterol diet model [32]. We also showed that Lr263 had consistent physiological activities on TG and TC content and fat composition under a hyperlipidemic model.

Table 2. Tissue weights at the end of the experiment.

\begin{tabular}{ccccccc}
\hline Organ Weight & Control & HCD & Lr263-1X & Lr263-2X & Lr263-5X & $\begin{array}{c}\text { Trend } \\
\text { Analysis }\end{array}$ \\
\hline Liver (g) & $3.50 \pm 0.37^{\mathrm{a}}$ & $5.97 \pm 0.28^{\mathrm{c}}$ & $5.47 \pm 0.32^{\mathrm{b}}$ & $5.47 \pm 0.37^{\mathrm{b}}$ & $5.35 \pm 0.31^{\mathrm{b}}$ & 0.0003 \\
Kidney (g) & $1.17 \pm 0.05$ & $1.14 \pm 0.05$ & $1.15 \pm 0.06$ & $1.17 \pm 0.08$ & $1.13 \pm 0.07$ & 0.8553 \\
Heart (g) & $0.53 \pm 0.04$ & $0.50 \pm 0.05$ & $0.52 \pm 0.06$ & $0.53 \pm 0.02$ & $0.50 \pm 0.03$ & 0.8219 \\
EFP (g) & $2.12 \pm 0.34^{\mathrm{a}}$ & $2.98 \pm 0.59^{\mathrm{b}}$ & $2.56 \pm 0.27^{\mathrm{ab}}$ & $2.40 \pm 0.49^{\mathrm{a}}$ & $2.41 \pm 0.40^{\mathrm{a}}$ & 0.0095 \\
Relative liver (\%) & $2.84 \pm 0.19^{\mathrm{a}}$ & $4.76 \pm 0.52^{\mathrm{c}}$ & $4.25 \pm 0.39^{\mathrm{b}}$ & $4.43 \pm 0.25^{\mathrm{bc}}$ & $4.48 \pm 0.43^{\mathrm{bc}}$ & 0.6720 \\
Relative kidney (\%) & $0.96 \pm 0.08$ & $0.91 \pm 0.07$ & $0.89 \pm 0.03$ & $0.95 \pm 0.07$ & $0.95 \pm 0.11$ & 0.3636 \\
Relative heart (\%) & $0.43 \pm 0.04$ & $0.40 \pm 0.05$ & $0.40 \pm 0.04$ & $0.43 \pm 0.02$ & $0.42 \pm 0.03$ & 0.0321 \\
Relative EFP (\%) & $1.71 \pm 0.16^{\mathrm{a}}$ & $2.38 \pm 0.56^{\mathrm{b}}$ & $1.98 \pm 0.19^{\mathrm{a}}$ & $1.92 \pm 0.25^{\mathrm{a}}$ & $2.01 \pm 0.28^{\mathrm{a}}$ & 0.1194 \\
\hline
\end{tabular}

Data are mean $\pm \mathrm{SD}, n=8$ hamsters per group. Values in the same row with different superscripts letters $(a, b, c)$ significantly differ at $p<0.05$ by one-way ANOVA. EFP: Epididymal fat pad.

\subsection{Effect of Lr263 Supplementation on Biochemical Analyses at the End of the Experiment}

In the present study, we observed the beneficial effects of Lr263 on indicators of lipid-lowering capacity. We further investigated whether six-week Lr263 treatment had any negative effect on other biochemical markers of hamsters. We examined the tissue- and health status-related biochemical parameters and major organs including liver, heart, kidney, and lung by histopathology (Table 3 and Figure 7). Supplementation of Lr263 for six weeks had no adverse effects. The ALT index significantly differed among groups $(\mathrm{F}(4,35)=7.168, p<0.05, \eta 2=0.450)$ and the HCD diet increased the ALT index $(p<0.0001)$ as compared with controls. For clinical application, statins, which are cholesterol-lowering drugs, affect all aspects of the cholesterol profile, but all have been shown to significantly elevate liver enzyme levels [33]. We found that Lr263 supplementation significantly decreased the ALT index and had dose-dependent effects on trend analysis ( $p=0.0143$ ). Therefore, Lr263 supplementation could provide alternative nutrient supplementation to ameliorate the side effects of statins and has a potential effect on lowering hyperlipidemia.

\subsection{Effect of Lr263 Supplementation on Histology at the End of the Experiment}

Liver tissue from hamsters fed a normal chow diet showed a clear hepatic cord and sinusoid (Figure 7). In a previous study, the high-fat diet-induced pathological morphology in livers significantly differed in rodent species. The fat was microvesicular in hamsters and mixed (macro- and microvesicular) in mice [34]. In the HCD-fed group, fatty liver changes (steatosis) were detected in all animals, with hepatocytes comprising microvesicles filled with small lipid droplets, which is similar with the previous pathological observation. The degree of fatty change was significantly lower in the Lr-263-5X than HCD-fed group, with no significant difference in steatosis status between Lr-263-1X, Lr-263-2X and HCD-fed groups. 
Table 3. Biochemical analysis of the L. reuteri 263 (Lr263) treatment groups at the end of experiment.

\begin{tabular}{ccccccc}
\hline Parameters & Control & HCD & Lr263-1X & Lr263-2X & Lr263-5X & Trend Analysis \\
\hline AST (U/L) & $48 \pm 10$ & $46 \pm 11$ & $45 \pm 8$ & $43 \pm 4$ & $44 \pm 4$ & 0.8777 \\
ALT (U/L) & $72 \pm 9^{\mathrm{a}}$ & $101 \pm 15^{\mathrm{c}}$ & $90 \pm 11^{\mathrm{bc}}$ & $89 \pm 12^{\mathrm{b}}$ & $87 \pm 5^{\mathrm{b}}$ & 0.0143 \\
LDH (U/L) & $139 \pm 25$ & $142 \pm 16$ & $137 \pm 16$ & $138 \pm 16$ & $134 \pm 13$ & 0.3844 \\
Albumin (g/dL) & $3.6 \pm 0.2$ & $3.5 \pm 0.2$ & $3.6 \pm 0.2$ & $3.6 \pm 0.2$ & $3.6 \pm 0.1$ & 0.8501 \\
TP (g/dL) & $5.6 \pm 0.3$ & $5.5 \pm 0.2$ & $5.8 \pm 0.2$ & $5.7 \pm 0.2$ & $5.6 \pm 0.2$ & 0.8501 \\
BUN (mg/dL) & $17.9 \pm 2.3$ & $17.8 \pm 1.8$ & $17.9 \pm 3.2$ & $17.6 \pm 0.8$ & $17.7 \pm 1.8$ & 0.7215 \\
Creatinine & $0.12 \pm 0.03$ & $0.12 \pm$ & $0.13 \pm 0.06$ & $0.12 \pm 0.04$ & $0.12 \pm 0.02$ & 0.6189 \\
(mg/dL) & 0.02 & & & \\
Glucose (mg/dL) & $99 \pm 12^{\mathrm{a}}$ & $151 \pm 25^{\mathrm{b}}$ & $110 \pm 17^{\mathrm{a}}$ & $103 \pm 21^{\mathrm{a}}$ & $98 \pm 14^{\mathrm{a}}$ & $<0.0001$ \\
\hline
\end{tabular}

Data are mean \pm SD for $n=8$ hamsters per group. Values in the same row with different superscripts letters $(\mathrm{a}, \mathrm{b}, \mathrm{c})$ significantly differ at $p<0.05$ by one-way ANOVA. AST, aspartate aminotransferase; ALT, alanine aminotransferase; LDH, lactate dehydrogenase; TP, total protein; BUN, blood urea nitrogen.
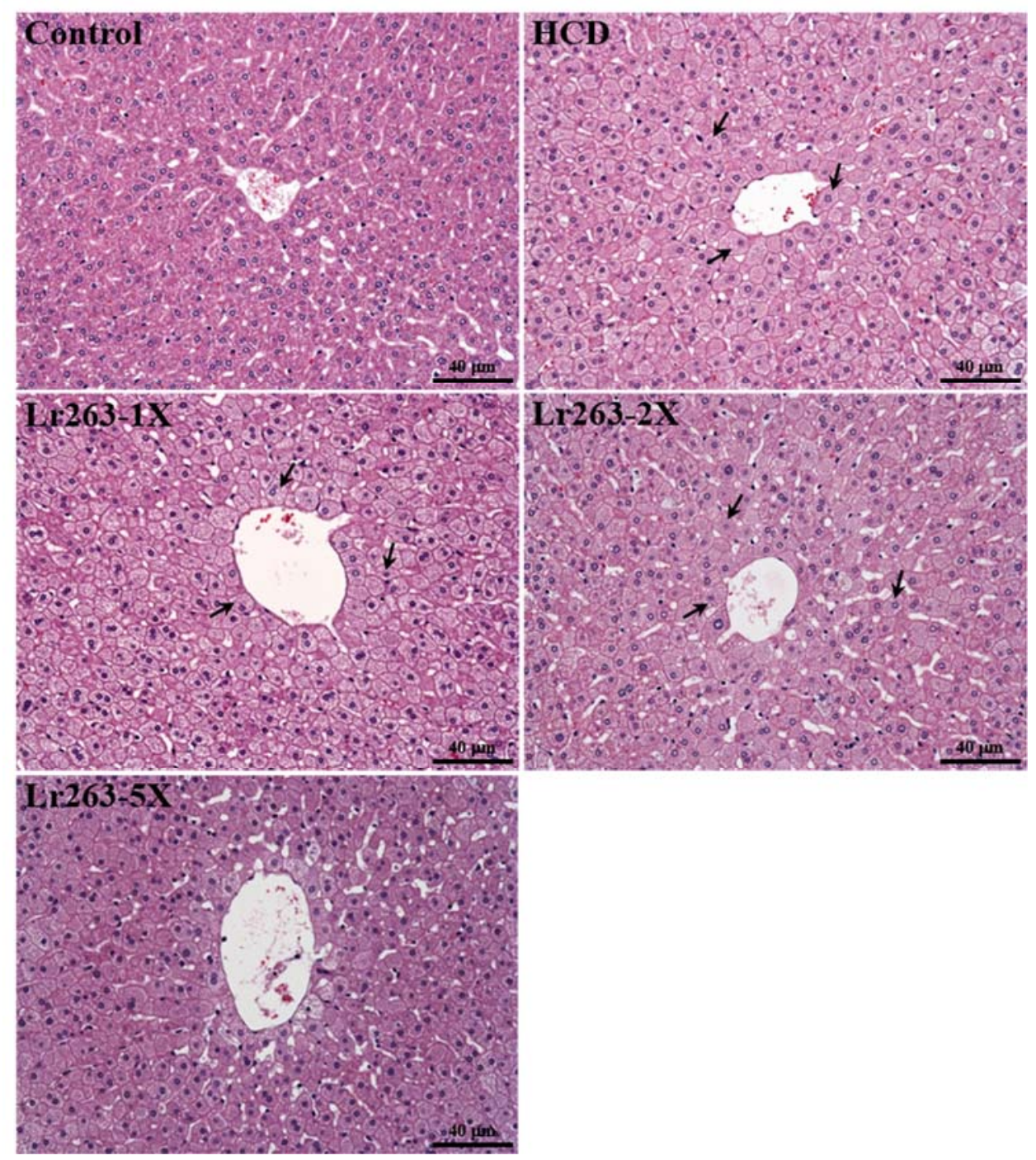

Figure 7. Effect of six-week supplementation with Lr263 on morphology of liver tissues in hyperlipidemic hamsters. Arrows indicate fat droplets. Specimens were photographed by light microscopy. (H \& E stain, magnification: $\times 200$, Scale bar, $40 \mu \mathrm{m}$ ) 


\section{Conclusions}

Lr263 has lipid-lowering actions by decreasing serum TG and TC levels, liver TG and TC levels, fecal TG levels and serum LDL-C and LDL-C/HDL-C levels in hyperlipidemic hamsters. We found that six-week Lr263 supplementation significantly improved the hyperlipidemia syndrome in hamsters. Lr263 increased HDL-C levels to decrease the LDL-C/HDL-C ratio, which is beneficial to human health by reducing the risk for developing cardiovascular disease. In biochemical study, we found no gross abnormalities attributed to Lr263 treatment. Many studies demonstrate the L. reuteri has antioxidant activity and immune functions [35,36]. The possible mechanism for reducing serum cholesterol by L. reuteri is activating bile salt hydrolase enzyme to increase bile acid excretion [37,38]. In clinical studies, L. reuteri significantly reduced LDL-C level and is considered an option to prevent cardiovascular disease [39]. In conclusion, our study provides experiment-based evidence to support that Lr263 may have potential as a therapeutic for reducing blood lipid levels and lowering hyperlipidemic effects.

\section{Acknowledgments}

This study was supported by a grant from the GenMont Biotech Inc., Tainan County, Taiwan. The authors are grateful to Chien-Chao Chiu for technical assistance in pathological examination. We also thank Laura Smales (BioMedEditing, Toronto, Canada) for her careful reading of the manuscript.

\section{Author Contributions}

Chi-Chang Huang and Hui-Yu Huang designed the experiments. Wen-Ching Huang, Yi-Ming Chen, Nai-Wen Kan, Chun-Sheng Ho, Wei Li, Ching-Hung Chan and Chi-Chang Huang carried out the laboratory experiments. Wen-Ching Huang, Yi-Ming Chen and Chi-Chang Huang analyzed the data, interpreted the results, prepared figures, and wrote the manuscript. Hui-Yu Huang and Chi-Chang Huang revised the manuscript. Nai-Wen Kan, Chun-Sheng Ho, Wei Li, Hui-Yu Huang and Chi-Chang Huang contributed reagents, materials and analysis platforms.

\section{Conflicts of Interest}

The authors declare no conflict of interest.

\section{References}

1. Tsai, T.Y.; Chu, L.H.; Lee, C.L.; Pan, T.M. Atherosclerosis-preventing activity of lactic acid bacteria-fermented milk soymilk supplemented with Momordica charantia. J. Agric. Food Chem. 2009, 57, 2065-2071.

2. Jones, M.L.; Martoni, C.J.; Parent, M.; Prakash, S. Cholesterol-lowering efficacy of a microencapsulated bile salt hydrolase-active Lactobacillus reuteri NCIMB 30242 yoghurt formulation in hypercholesterolaemic adults. Br. J. Nutr. 2012, 107, 1505-1513.

3. Ljungh, A.; Wadström, T. Lactobacillus Molecular Biology: From Genomics to Probiotics. Caister Academic Press: Norfolk, England, 2009. 
4. Chen, Y.M.; Shih, T.W.; Chiu, C.P.; Pan, T.M.; Tsai, T.Y. Effects of lactic acid bacteria-fermented soy milk on melanogenesis in B16F0 melanocytes. J. Funct. Foods 2013, 5, 395-405.

5. Noohi, N.; Ebrahimipour, G.; Rohani, M.; Talebi, M.; Pourshafie, M.R. Phenotypic characteristics and probiotic potentials of Lactobacillus spp. isolated from poultry. Jundishapur J. Microbiol. 2014, 7, e17824, doi:10.5812/jjm.17824.

6. Talarico, T.L.; Casas, I.A.; Chung, T.C.; Dobrogosz, W.J. Production and isolation of reuterin, a growth inhibitor produced by Lactobacillus reuteri. Antimicrob Agents Chemother. 1988, 32, 1854-1858.

7. Ojetti, V.; Ianiro, G.; Tortora, A.; D’Angelo, G.; di Rienzo, T.A.; Bibbò, S.; Gasbarrini, A. The effect of Lactobacillus reuteri supplementation in adults with chronic functional constipation: A randomized, double-blind, placebo-controlled trial. J. Gastrointestin. Liver Dis. 2014, 23, 387-391.

8. Holz, C.; Busjahn, A.; Mehling, H.; Arya, S.; Boettner, M.; Habibi, H.; Lang, C. Significant Reduction in Helicobacter pylori Load in Humans with Non-viable Lactobacillus reuteri DSM17648: A Pilot Study. Probiotics Antimicrob. Proteins 2014, doi:10.1007/s12602-014-9181-3.

9. Sima, A.; Bulla, A.; Simionescu, N. Experimental obstructive coronary atherosclerosis in the hyperlipidemic hamster. J. Submicrosc. Cytol. Pathol. 1990, 22, 1-16.

10. Sullivan, M.P.; Cerda, J.J.; Robbins, F.L.; Burgin, C.W. The gerbil, hamster, and guinea pig as rodent models for hyperlipidemia. Lab. Anim. Sci. 1993, 43, 575-578.

11. Stancu, C.S.; Sanda, G.M.; Deleanu, M.; Sima, A.V. Probiotics determine hypolipidemic and antioxidant effects in hyperlipidemic hamsters. Mol. Nutr. Food. Res. 2014, 58, 559-568.

12. Qiao, Y.; Sun, J.; Xia, S.; Li, L.; Li, Y.; Wang, P.; Shi, Y.; Le, G. Effects of different Lactobacillus reuteri on inflammatory and fat storage in high-fat diet-induced obesity mice model. J. Funct. Foods 2015, 14, 424-434.

13. Singh, T.P.; Malik, R.K.; Katkamwar, S.G.; Kaur, G. Hypocholesterolemic effects of Lactobacillus reuteri LR6 in rats fed on high-cholesterol diet. Int. J. Food Sci. Nutr. 2015, 66, 71-75.

14. Chen, W.C.; Huang, W.C.; Chiu, C.C.; Chang, Y.K.; Huang, C.C. Whey protein improves exercise performance and biochemical profiles in trained mice. Med. Sci. Sports Exerc. 2014, 46, 1517-1524.

15. Yang, L.; Leung, K.Y.; Cao, Y.; Huang, Y.; Ratnayake, W.M.; Chen, Z.Y. Alpha-linolenic acid but not conjugated linolenic acid is hypocholesterolaemic in hamsters. Br. J. Nutr. 2005, 93, 433-438.

16. Liu, X.M.; Wu, F.H. Comparison of animal models of hyperlipidemia. Zhong Xi Yi Jie He Xue Bao 2004, 2, 132-134.

17. Zhang, Z.; Wang, H.; Jiao, R.; Peng, C.; Wong, Y.M.; Yeung, V.S.; Huang, Y.; Chen, Z.Y. Choosing hamsters but not rats as a model for studying plasma cholesterol-lowering activity of functional foods. Mol. Nutr. Food Res. 2009, 53, 921-930.

18. Gao, Y.; Li, K.; Tang, S.; Xiao, Y. Study on animal models for hyperlipidemia. Wei Sheng Yan Jiu 2002, 31, 97-99.

19. Lee, C.L.; Tsai, T.Y.; Wang, J.J.; Pan, T.M. In vivo hypolipidemic effects and safety of low dosage Monascus powder in a hamster model of hyperlipidemia. Appl. Microbiol. Biotechnol. 2006, $70,533-540$.

20. Lee, C.L.; Hung, H.K.; Wang, J.J.; Pan, T.M. Red mold dioscorea has greater hypolipidemic and antiatherosclerotic effect than traditional red mold rice and unfermented dioscorea in hamsters. J. Agric. Food Chem. 2007, 55, 7162-7169. 
21. Chen, W.; Fan, S.; Xie, X.; Xue, N.; Jin, X.; Wang, L. Novel PPAR pan agonist, ZBH ameliorates hyperlipidemia and insulin resistance in high fat diet induced hyperlipidemic hamster. PLOS ONE. 2014, 9, e96056, doi:10.1371/journal.pone.0096056.

22. Yonejima, Y.; Ushida, K.; Mori, Y. Effect of lactic acid bacteria on lipid metabolism and fat synthesis in mice fed a high-fat diet. Biosci. Microbiota Food Health 2013, 32, 51-58.

23. Pereira, D.I.; Gibson, G.R. Effects of consumption of probiotics and prebiotics on serum lipid levels in humans. Crit. Rev. Biochem. Mol. Biol. 2002, 37, 259-281.

24. Taranto, M.P.; Medici, M.; Perdigon, G.; Ruiz Holgado, A.P.; Valdez, G.F. Effect of Lactobacillus reuteri on the prevention of hypercholesterolemia in mice. J. Dairy Sci. 2000, 83, 401-403.

25. Barona, J.; Fernandez, M.L. Dietary cholesterol affects plasma lipid levels, the intravascular processing of lipoproteins and reverse cholesterol transport without increasing the risk for heart disease. Nutrients 2012, 4, 1015-1025.

26. Taranto, M.P.; Fernandez Murga, M.L.; Lorca, G.; de Valdez, G.F. Bile salts and cholesterol induce changes in the lipid cell membrane of Lactobacillus reuteri. J. Appl. Microbiol. 2003, 95, 86-91.

27. Begley, M.; Hill, C.; Gahan, C.G. Bile salt hydrolase activity in probiotics. Appl. Environ. Microbiol. 2006, 72, 1729-1738.

28. Tsai, C.C.; Lin, P.P.; Hsieh, Y.M.; Zhang, Z.Y.; Wu, H.C.; Huang, C.C. Cholesterol-lowering potentials of lactic acid bacteria based on bile-salt hydrolase activity and effect of potent strains on cholesterol metabolism in vitro and in vivo. Sci. World J. 2014, doi:10.1155/2014/690752.

29. Friedman, M.; Fitch, T.E.; Levin, C.E.; Yokoyama, W.H. Feeding tomatoes to hamsters reduces their plasma low-density lipoprotein cholesterol and triglycerides. J. Food Sci. 2000, 65, 897-900.

30. Mencarelli, A.; Cipriani, S.; Renga, B.; Bruno, A.; D’Amore, C.; Distrutti, E.; Fiorucci, S. VSL\#3 resets insulin signaling and protects against NASH and atherosclerosis in a model of genetic dyslipidemia and intestinal inflammation. PLoS One. 2012, 7, e45425, doi:10.1371/journal. pone.0045425.

31. Kobayashi, M.; Egusa, S.; Fukuda, M. Isoflavone and protein constituents of lactic acid-fermented soy milk combine to prevent dyslipidemia in rats fed a high cholesterol diet. Nutrients 2014, 6, 5704-5723.

32. Jo, S.Y.; Choi, E.A.; Lee, J.J.; Chang, H.C. Characterization of starter kimchi fermented with Leuconostoc kimchii GJ2 and its cholesterol-lowering effects in rats fed a high-fat and high-cholesterol diet. J. Sci. Food Agric. 2014, doi:10.1002/jsfa.7018.

33. Chawda, H.M.; Mandavia, D.R.; Parmar, P.H.; Baxi, S.N.; Tripathi, C.R. Hypolipidemic activity of a hydroalcoholic extract of Cyperus scariosus Linn. root in guinea pigs fed with a high cholesterol diet. Chin. J. Nat. Med. 2014, 12, 819-826.

34. Gilat, T.; Leikin-Frenkel, A.; Goldiner, I.; Juhel, C.; Lafont, H.; Gobbi, D.; Konikoff, F.M. Prevention of diet-induced fatty liver in experimental animals by the oral administration of a fatty acid bile acid conjugate (FABAC). Hepatology 2003, 38, 436-442.

35. Hathout, A.S.; Mohamed, S.R.; El-Nekeety, A.A.; Hassan, N.S.; Aly, S.E.; Abdel-Wahhab, M.A. Ability of Lactobacillus casei and Lactobacillus reuteri to protect against oxidative stress in rats fed aflatoxins-contaminated diet. Toxicon 2011, 58, 179-186.

36. Forsberg, A.; Abrahamsson, T.R.; Björkstén, B.; Jenmalm, M.C. Pre-and post-natal Lactobacillus reuteri supplementation decreases allergen responsiveness in infancy. Clin. Exp. Allergy 2013, $43,434-442$. 
37. Martoni, C.; Bhathena, J.; Jones, M.L. Investigation of microencapsulated BSH active Lactobacillus in the simulated human GI tract. J. Biomed. Biotechnol. 2007, 2007, 1-9.

38. Ooi, L.G.; Ahmad, R.; Yuen, K.H. Lactobacillus acidophilus CHO-220 and inulin reduced plasma total cholesterol and low-density lipoprotein cholesterol via alteration of lipid transporters. J. Dairy. Sci. 2010, 93, 5048-5058.

39. Jones, M.L.; Martoni, C.J.; Prakash, S. Cholesterol lowering and inhibition of sterol absorption by Lactobacillus reuteri NCIMB 30242: A randomized controlled trial. Eur. J. Clin. Nutr. 2012, 66, 1234-1241.

(C) 2015 by the authors; licensee MDPI, Basel, Switzerland. This article is an open access article distributed under the terms and conditions of the Creative Commons Attribution license (http://creativecommons.org/licenses/by/4.0/). 